\title{
The relationship between sagittal spinal curvatures and isokinetic trunk muscle endurance after aquatic exercise training in survivors of breast cancer
}

\author{
JUSTYNA MARIA HANUSZKIEWICZ*, MAREK WOŹNIEWSKI, IWONA MALICKA \\ Department of Rehabilitation in Internal Diseases, Faculty of Physiotherapy, \\ University School of Physical Education in Wrocław, Wrocław, Poland.
}

\begin{abstract}
Purpose: Assessment of the impact of aquatic exercises on the relationship between sagittal spinal curvatures and isokinetic trunk muscle endurance against standard land exercises in women after breast cancer treatment. Methods: 40 women post breast cancer divided into two groups: a study group $(n=20)$ participated in the aquatic exercises and a control group $(n=20)$ performed the land exercises. Data collection occurred both before and after the 8 week intervention. Measurements were taken from the SG and CG performing antagonistic movements (flexions/extensions) to assess the endurance $(120 \% \mathrm{~s})$ in isokinetic conditions and a photogrammetric examination to define sagittal spinal curvatures. Results: A significant improvement $(p<0.05)$ in total work and average power was found among women after the aquatic exercises irrespective of the muscle group under examination. There were no significant changes in any examined parameters after the land exercises. In the study group, there was a significant association of gamma angle decrease and an increase of all examined functional parameters of total work and average power of both the extensor muscles $(r=-0,49$ to -0.51$)$ and the trunk flexors $(r=-0.48$ to -0.51$)$. In the control group, a similar tendency of functional-postural changes was observed only for the extensor muscles of the trunk ( $r=-0.54$ to -0.58$)$. Conclusions: General exercises performed in water, as opposed to those performed on land, are more effective in terms of the functional-postural retations in women after breast cancer.
\end{abstract}

Key words: physical activity, body posture, isokinetics, photogrammetry, strength deficit

\section{Introduction}

Breast cancer $(\mathrm{BC})$ is the most common form of cancer in women in the world. The adverse side effects of breast cancer treatment relate to both musculoskeletal and nervous systems, thereby affecting the functioning of the posture regulation system. The emerging restrictions on joint mobility, contractures, pain, muscle weakness, tissue fibrosis, peripheral nerve injuries, as well as disturbances in the psychological sphere are the main causes of adverse changes in body posture and trunk balance of women treated for BC [13]. These changes affect the spine's normal ability to transfer loads [2], [12].
The trunk's optimal muscle function plays a key role in maintaining the physiological curvature of the spine by ensuring its stability and preventing diseases and excessive strain on the spine. Changes in the spine's geometry affect the performance of spinal muscles. Excessive sagittal curvatures may move the trunk load away from the line of gravity, which disrupts the balanced spinal moments. An increase in the trunk muscle function is required to balance the distorted spinal torque, which results in excessive spinal strain [13].

On the other hand, diminished anteroposterior curvatures lead to a shortening of the internal moment arm of the trunk extensors. A change in the lever increases the demand for strength while interfering with

\footnotetext{
* Corresponding author: Justyna Hanuszkiewicz, The Faculty of Physiotherapy, University School of Physical Education in Wrocław, al. Paderewskiego 35, 51-612, Wrocław, Poland. Phone: +48713473519, e-mail: justyna.hanuszkiewicz@awf.wroc.pl

Received: October 2nd, 2019

Accepted for publication: December 12th, 2019
} 
the muscular work of the trunk extensors, thus, affecting the amount of compression and generating a greater load on the spine [12].

The biomechanical aspects of the trunk's postural and functional determinants give rise to an interesting issue of the mutual relationship between the body posture and the strength of the aforementioned muscle group [3], [12]. Achieving and maintaining a particular posture is based on the continuously alternating contraction of muscle groups, which, at the same time, does not require a lot of muscular effort, indicating the endurance nature of postural muscles [2]. It has demonstrated that a properly selected exercise programme guarantees an improvement in physical function [6], [15]. Physical rehabilitation in the aquatic environment provides a specific type of resistance workout. The body is exposed to a number of mechanical and thermal factors during aquatic exercises, above all those resulting from the water's properties such as buoyancy, hydrostatic pressure, resistance, viscosity, and wave, which result in effects that can be local, segmental or general [15].

Aquatic exercises (AE) are increasingly used for patients rehabilitating after various disease entities [1], [16] and they are also a popular form of physical activity among elderly people [5], [24]. Studies in this area show a significant improvement in cardiorespiratory parameters, body composition and muscle strength [24] as well as an improvement of neuromuscular function [25]. It was proved that exercises in water environment are also an effective form of rehabilitation of cancer patients. The 8-week supervised water physical therapy focused on low-intensity exercise was effective for improving neck and shoulder pain and reducing the presence of trigger points in $\mathrm{BC}$ survivors, compared to usual care [9]. Moreover, Contarero-Villanueva et al. [8] found that the 8-week supervised AE programme in deep water decreased fatigue and improved leg-abdominal muscle endurance and several aspects of mood state such as tension, depression and anger in $\mathrm{BC}$ survivors suffering moderate cancer-related fatigue (CRF). The study [8] concurs with the results of multimodal programme incorporating deep water running for improving cancer-related fatigue and quality of life in BC survivors [10]. In addition, it was reported that hydrotherapy may reduce the pain threshold, waist circumference [7] and lymphedema [18] in BC survivors.

Although it is now widely accepted that the aquatic environment increases positive muscle function [8], [9] and improves overall body condition [7], [10], [18], it has not been established whether the AE affect the relationship between sagittal spinal curva- tures and isokinetic trunk muscle endurance in women after BC. As such, conducting research on mutual functional-postural relationships as important indicators of both diagnostic values of musculoskeletal dysfunctions as well as determining the plan and goals of rehabilitation among survivors of $\mathrm{BC}$ is necessary. We hipothesized that water physical therapy due to the physicochemical properties of the environment could demonstrate a higher efficiency of the rehabilitation process than standard land exercises (LE). Therefore, the aim of the study was to examine the effect of aquatic exercises on the relationship between sagittal spinal curvatures and isokinetic trunk muscle endurance against standard land exercises in women after breast cancer treatment.

\section{Materials and methods}

\subsection{Participants}

To participate in the study, women were qualified by a physiotherapist under the supervision of a physician. An initial sample consisted of 60 potentially eligible participants if they (a) had a diagnosis of BC, (b) were between 50 and 75 years of age, (c) had finished co-adjuvant treatment, (d) did not have active cancer, (e) did not have lymphedema within the upper extremity.

Participants were excluded if they were taking any medication at the time of the study or they had physical limitations associated with mental, orthopedic, cardiological, neurological and oncological conditions (for the oncology group absence of cancers other than BC).

Following screening for inclusion and exclusion and confirmation for eligibility by the physiotherapist, the baseline sample included 40 participants. Participants $(n=40)$ were divided into two groups with 20 women in each group. The study group (SG) performed the AE programme, while the control group (CG) executed standard LE programme. This was a randomized controlled trial. Allocation was concealed via sealed opaque envelopes.

The average age of women taking part in the LE programme totalled 59.4 years $( \pm 7.47)$, average body height $1.59 \mathrm{~m}( \pm 0.57)$, and average body mass $74.1 \mathrm{~kg}$ $( \pm 10.23)$. A breast conserving therapy was applied to $20 \%$ of examined, and modified radical mastectomy to $80 \%$ of women. Adjuvant therapy involved $90 \%$ of examined; including chemotherapy $-70 \%$, radiotherapy $-45 \%$, and hormone therapy $-55 \%$ of women. The average time since the surgery was 6.1 years $( \pm 4.63)$. 
In turn, the average age of women participating in the AE program was 63.0 years $( \pm 7.58)$, average body height $1.61 \mathrm{~m}( \pm 0.55)$, and average body mass $74.7 \mathrm{~kg}$ $( \pm 10.67)$. In $65 \%$ of the subjects, a radical mastectomy was performed, while in $35 \%$ the breast conserving therapy. Adjuvant therapy was used in $90 \%$ of subjects in the form of radiotherapy $-50 \%$ of examined, chemotherapy $-45 \%$ of examined and hormone therapy $-50 \%$ of examined. The average time since the surgery was 7.2 years $( \pm 6.41)$. The intergroup comparison of somatic parameters and the time since surgery did not show statistically significant differences $(p<0.05)$. Similarly, an analysis of the treatment methods applied showed no statistically significant differences between the groups $(p<0.05)$.

The study was approved by the Local Bioethics Committee of the University of Physical Education in Wrocław. The participants received oral and written information about the study and signed an informed and voluntary consent for participation. The trial was registered with the Australian and New Zealand Clinical Trials Registry (ANZCTR \#: 12618002017202).

\subsection{Functional assessment of the trunk muscles}

The strength-velocity parameters of the erector spinae (prime mover for the trunk flexion) and rectus abdominis (prime mover for the trunk extension) [14] were measured using the Biodex Multi-Joint 3 Isokinetic Dynamometer. Measurements of the trunk's alternating flexions and extensions with a maximum force at a selected angular velocity were recorded. The velocity of $120^{\circ} / \mathrm{s}$ was selected as it is considered to be the most suitable measures endurance and that faster speeds are usually performed for 10 to 20 repetitions [3], [6], [21]. Therefore, each woman being examined performed 20 consecutive repetitions of sagittal trunk extension and flexion without interruption.

The examined participant was placed in a semistanding position in a chair in a way that the axis of rotation of the joint and the dynamometer were aligned. The seat and the dynamometer were adjusted for each woman before testing so that the lower cushion of the dynamometer was at the height of L5/S1 spine segment. For the purpose of isolating the movement, the position was stabilized by means of belts and appropriate regulation, and it was adjusted to the needs of each examined participant. The starting position began with a maximum extension of the trunk. The women were told to complete the extensions and flexions using their maximum strength as fast as possible (Fig. 1).

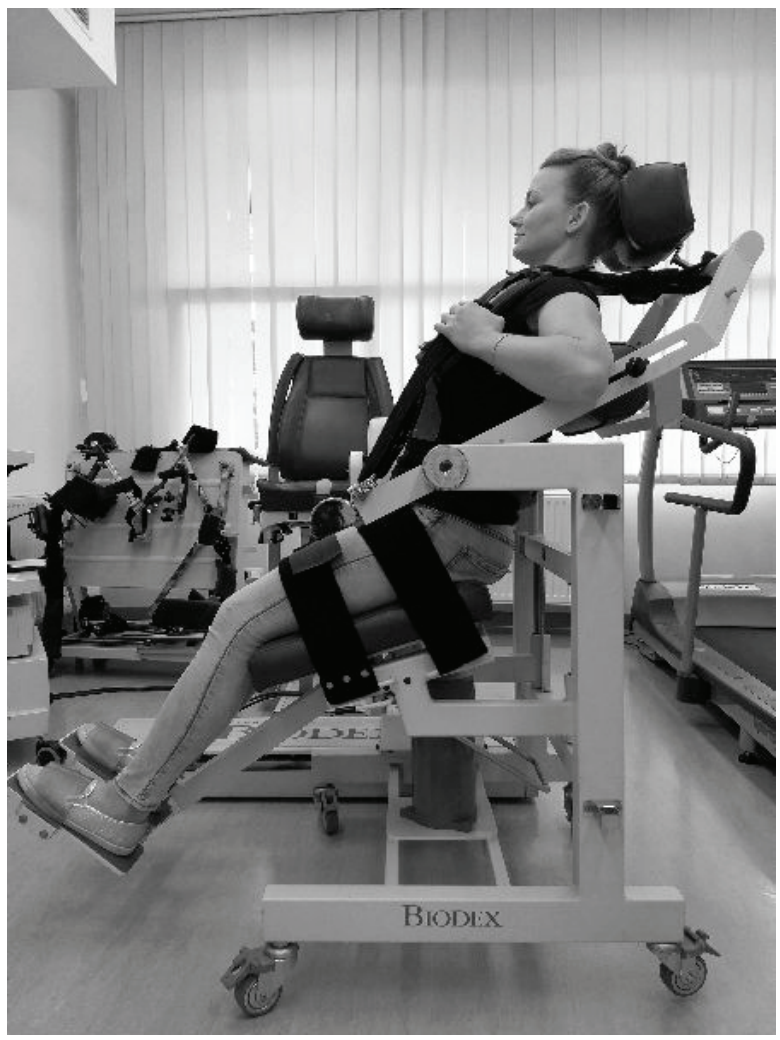

Fig. 1. Isokinetic examination of the trunk muscles: semi-standing position and stabilization of the body (source: own material)

The same range of the trunk flexion and extension was set around $70^{\circ}\left(20^{\circ}\right.$ - extension, $50^{\circ}$ - flexion $)$ for all women, and it was kept constant in order to standardise the intergroup comparisons. It was set up in such way that all of the participants could perform it in the same manner without reporting pain. Therefore, the range of motion was deliberately limited, in order to avoid stressful situations arising out of fear of pain. The test was preceded by a verbal instruction, and it consisted of a warm-up (several test runs) and a proper part.

Based on the existing examinations using isokinetic tests [3], [12], [21], the following parameters for endurance measurements of the spine muscles were selected:

$>\mathrm{TW}(\mathrm{J})$ - Total Work;

$>\mathrm{AP}(\mathrm{W})-$ Average Power.

\subsection{Sagittal spinal curvatures examination}

A postural examination using a photogrammetric method was performed on all participants. It is con- 
sidered to be noninvasive, safe method and has good reliability as well as concurrent validity with radiographic measures in determining the magnitude of the Cobb angle and lumbar lordosis [4]. Studies included an evaluation of the anterior-posterior curvatures of the spine, which was based on direct observation of the subject using a camera. The measurements took place in several stages as follows:

$>$ on the body of the participant, the following were marked: spinous processes from $\mathrm{C} 7$ to $\mathrm{S} 1$, inferior angles of the scapulas, posterior superior iliac spines and acromions;

the participant stood facing the camera backwards at a distance of $2.6 \mathrm{~m}$ so that the image of her trunk was visible on the computer screen. Each woman being examined adopted a relaxed, unconstrained standing position looking straight ahead, with upper limbs hanging down freely along the trunk, lower limbs upright in the knee joints with the same distribution of body weight, with feet parallel to each other and heels in a line;

next, the examined participant's spatial record of the trunk image was taken in the computer memory.

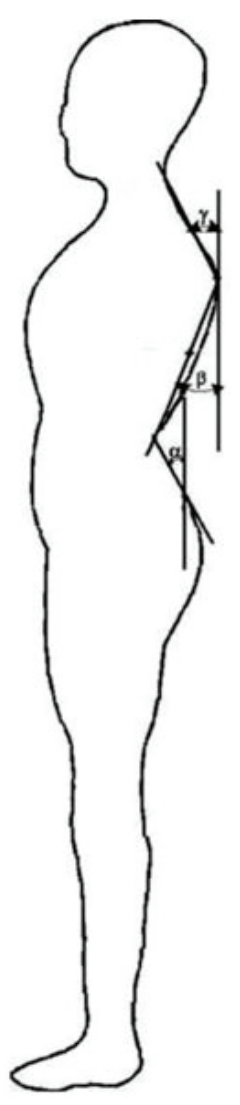

Fig. 2. Angular parameters of the sagittal spinal curvatures (source: own material).

Abverations: $\alpha$ - alfa angle (lumbosacral angle);

$\beta$ - beta angle (thoracolumbar angle);

$\gamma$-gamma angle (upper thoracic angle)
The parameters determining sagittal spinal curvatures were selected for analysis based on the literature on computer diagnostics of body posture [3], [4]. The following angular parameters were evaluated in detail (Fig. 2):

$>$ alpha angle $\left[{ }^{\circ}\right]$ - inclination of the lumbosacral section;

beta angle $\left[{ }^{\circ}\right]$ - inclination of the thoracolumbar section;

gamma angle $\left[^{\circ}\right]$ - inclination of the upper thoracic section.

\subsection{Training intervention}

The exercise programmes were based on previous guidelines for cancer survivors [22]. The programmes objectives were to improve health status with minimal risk of injury. This supervised programmes consisted of 45-minute sessions, 2 times a week, over 8 weeks. The programmes were conducted by a physiotherapist. The CG trained in gymnastic hall with a temperature of $22-24{ }^{\circ} \mathrm{C}$ and a wooden floor. The SG exercised in a swimming pool with a water temperature of $30-32{ }^{\circ} \mathrm{C}$ and depth of $0.8 \mathrm{~m}$ with unrestricted arm movements above the water. Exercises were based on organization of individual training units in a similar manner and exercises performance in standing, kneeling and lying positions (Table 1).

The basic parameter defining the intensity of physical exercise was the pulse limit [8]. Heart rate monitoring was combined with time options using the heart pulsometers manufactured by Polar, to determine the heart rate zone within which the physical effort was exerted. The intensity of exercises was at a similar level of $70-75 \%$ of maximum heart rate, determined individually for each subject using a formula of 220-age.

In both groups, the additional criterion for inclusion in the study was at least an $80 \%$ attendance for each participant.

\subsection{Statistical analyses}

Results were analysed using Statistica 13.0 from StatSoft. The normality of distribution was verified using the Shapiro-Wilk test, with findings that the hypothesis of normal distribution was not rejected.

Pearson $\chi^{2}$ test was performed to analyze discrete properties relating to cancer treatment within the two groups of physical activity. Comparison of the somatic characteristics and the time from surgery in both groups was performed using the Student's $t$-test. 
Table 1. Land and aquatic exercise programmes

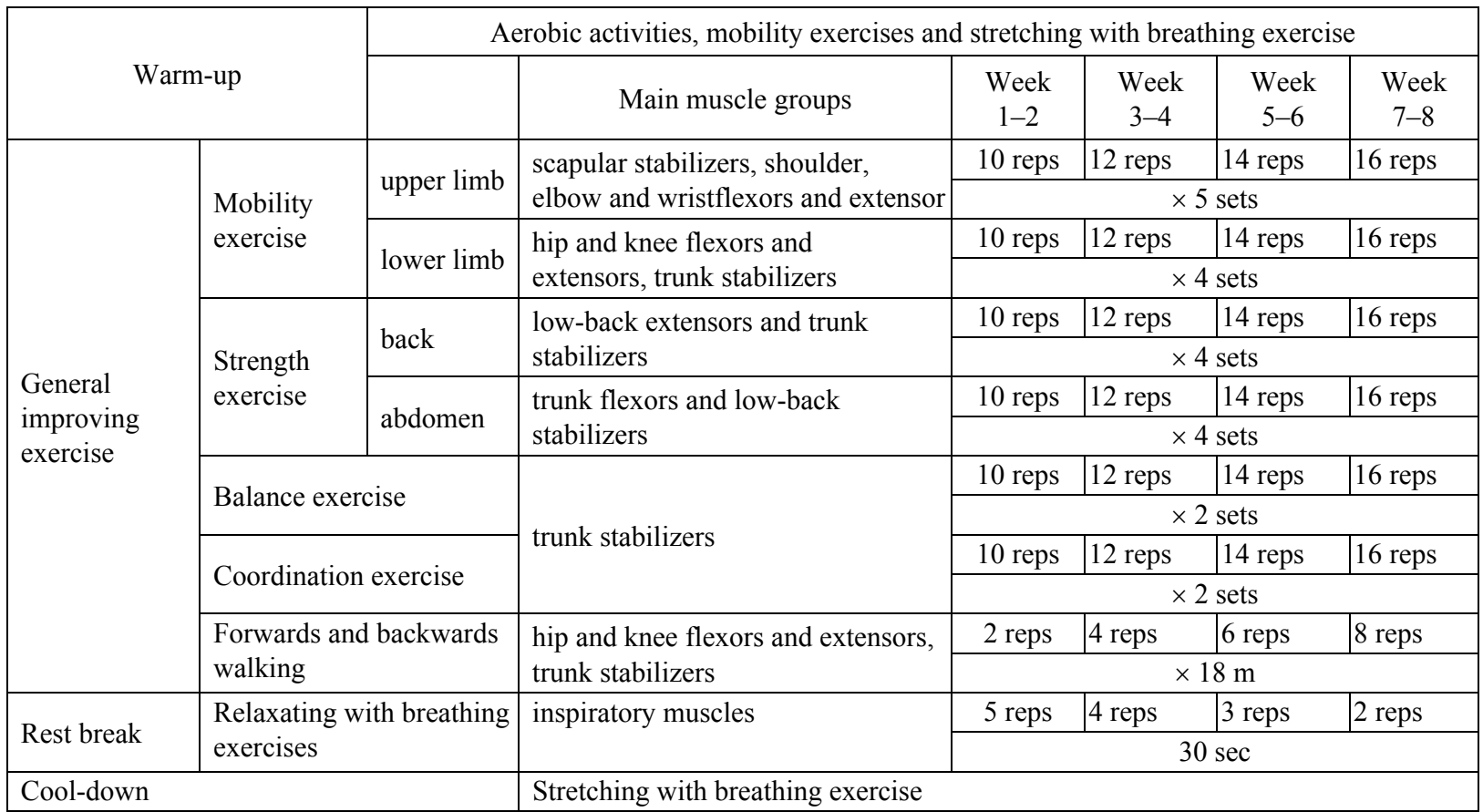

The two-way analysis of variance ANOVA (with the Scheffe post-hoc test) with time (pre- and post-intervention) as the within-subject variable and intervention (Aquatic-Control) as the between subjects variable was used to examine the effects of the intervention on sagittal spinal curvatures and isokinetic trunk muscle endurance.

Next, the Pearson's correlation coefficient was calculated to determine the strength of the relationship between the trunk muscle function and body posture within the two groups. All values of tests and coefficients were considered statistically significant at $p<0.05$.

\section{Results}

The two-way ANOVA was used to examine the effects of the intervention on the strength-velocity (Table 2) and the body posture parameters (Table 3).
Detailed parameters describing sagittal spinal curvatures and trunk muscle function in the examined groups are presented in Figs. 3-5. The intergroup comparison of examined parameters showed a statistically significant improvement in TW and AP among women after the $\mathrm{AE}$ programme irrespective of the muscle group under investigation. There were no significant changes in any examined parameters in response to the LE programme (Figs. 3-5).

To perform the statistical analysis, it was important to assess the group $\mathrm{x}$ study interaction. The ANOVA revealed significant interactions for TW of the trunk extensor muscles in SG $(p<0.001)$ and for TW of the trunk flexor muscles in both CG $(p<0.05)$ and SG $(p<$ $0.001)$. Similarly, the significant interaction effects were observed for AP of the trunk extensor muscles in SG $(p<0.001)$ and for AP of the trunk flexor muscles in both CG $(p<0.05)$ and SG $(p<0.001)$. In the case of the body posture parameters the group $\mathrm{x}$ study interaction was recorded for only the beta angle in SG $(p<0.001)$.

Table 2. The $p$-value of the two-way ANOVA results for the strength-velocity parameters

\begin{tabular}{|c|c|c|c|c|}
\hline \multirow{2}{*}{\multicolumn{2}{|c|}{ Strength-velocity parameters }} & \multicolumn{3}{|c|}{$p$-value } \\
\hline & & \multirow{2}{*}{$\frac{\text { Group }}{<0.001^{*}}$} & \multirow{2}{*}{$\begin{array}{c}\text { Study } \\
<0.001^{*}\end{array}$} & \multirow{2}{*}{$\begin{array}{c}\begin{array}{c}\text { Group x study } \\
\text { interaction }\end{array} \\
<0.01^{*}\end{array}$} \\
\hline \multirow{2}{*}{ Total Work [J] } & $\mathrm{E}$ & & & \\
\hline & $\mathrm{F}$ & $<0.01 *$ & $<0.001 *$ & $<0.05^{*}$ \\
\hline \multirow{2}{*}{ Average Power [W] } & $\mathrm{E}$ & $<0.01 *$ & $<0.001 *$ & $<0.01 *$ \\
\hline & $\mathrm{F}$ & $<0.001 *$ & $<0.001^{*}$ & $<0.01^{*}$ \\
\hline
\end{tabular}

Abbverations: $\mathrm{E}$ - trunk extensors; F - trunk flexors; * denotes significances. 
Table 3. The $p$-value of the two-way ANOVA results for the body posture parameters

\begin{tabular}{|l|c|c|c|}
\hline \multirow{2}{*}{$\begin{array}{c}\text { Body posture } \\
\text { parameters }\end{array}$} & \multicolumn{3}{|c|}{$p$-value } \\
\cline { 2 - 4 } & Group & Study & $\begin{array}{c}\text { Group x study } \\
\text { interaction }\end{array}$ \\
\hline Alfa $\left[^{\circ}\right]$ & 0.64 & 0.11 & 0.14 \\
\hline Beta $\left[^{\circ}\right]$ & $<0.05^{*}$ & $<0.001^{*}$ & $<0.01^{*}$ \\
\hline Gamma $\left[^{\circ}\right]$ & 0.17 & 0.60 & 0.74 \\
\hline
\end{tabular}

Abverations: Alfa - lumbosacral angle, Beta - thoracolumbar angle, Gamma - upper thoracic angle.

* denotes significances.
Next, the Pearson's correlation coefficient was calculated. For the SG, before commencement of exercises, a statistically significant directly proportional relationship between the alpha angle and TW $(r=0.49)$ and AP $(r=0.47)$ of the trunk extensor muscles was observed. In response to the AE programme, the value of the gamma angle has been decreased together with an increase in TW $(r=-0.48)$ and AP $(r=-0.51)$ for the trunk flexor muscles and a correspondingly important relationship $(r=-0.49, r=-0.51)$ for the trunk extensor muscles (Table 4).

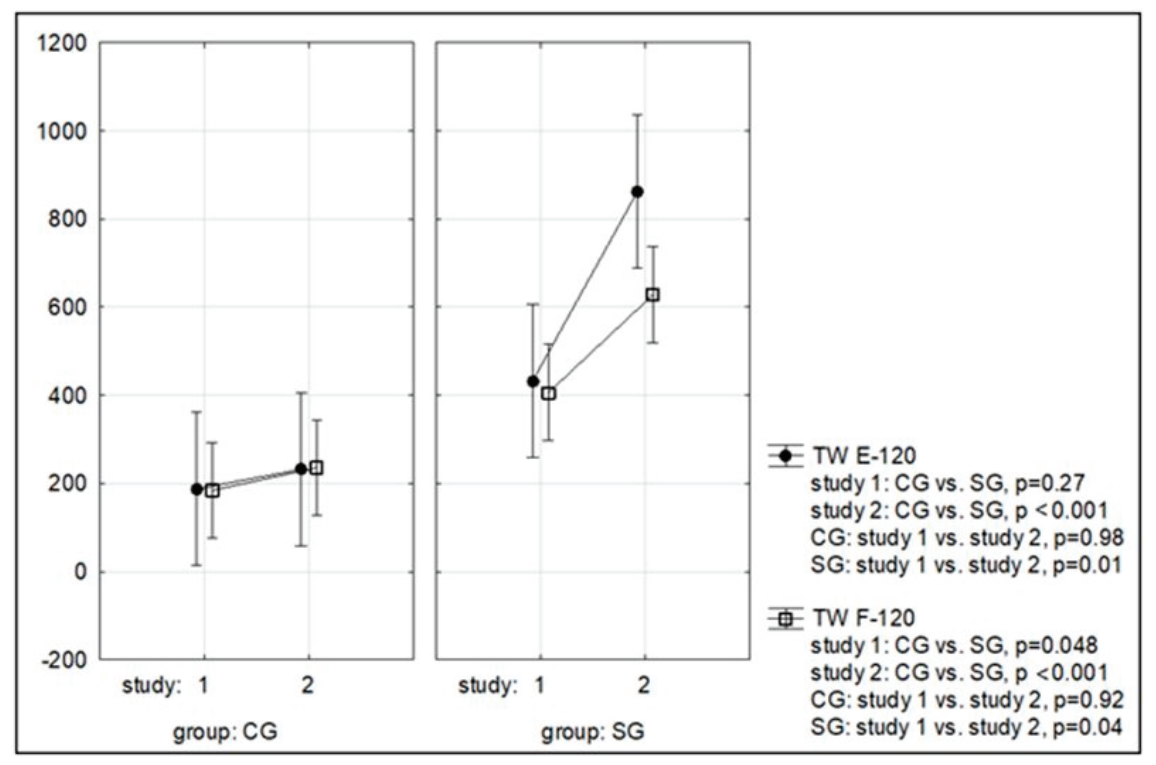

Fig. 3. Total Work $[\mathrm{J}]$ of the trunk muscles before and after the 8 -week exercise programmes in the examined groups.

Values are expressed as mean (95\% confidence interval), $p$-values based on the Scheffe post-hoc test.

Abverations: CG - Control Group, SG - Study Group, TW - Total Work, E - trunk extensors, F - trunk flexors

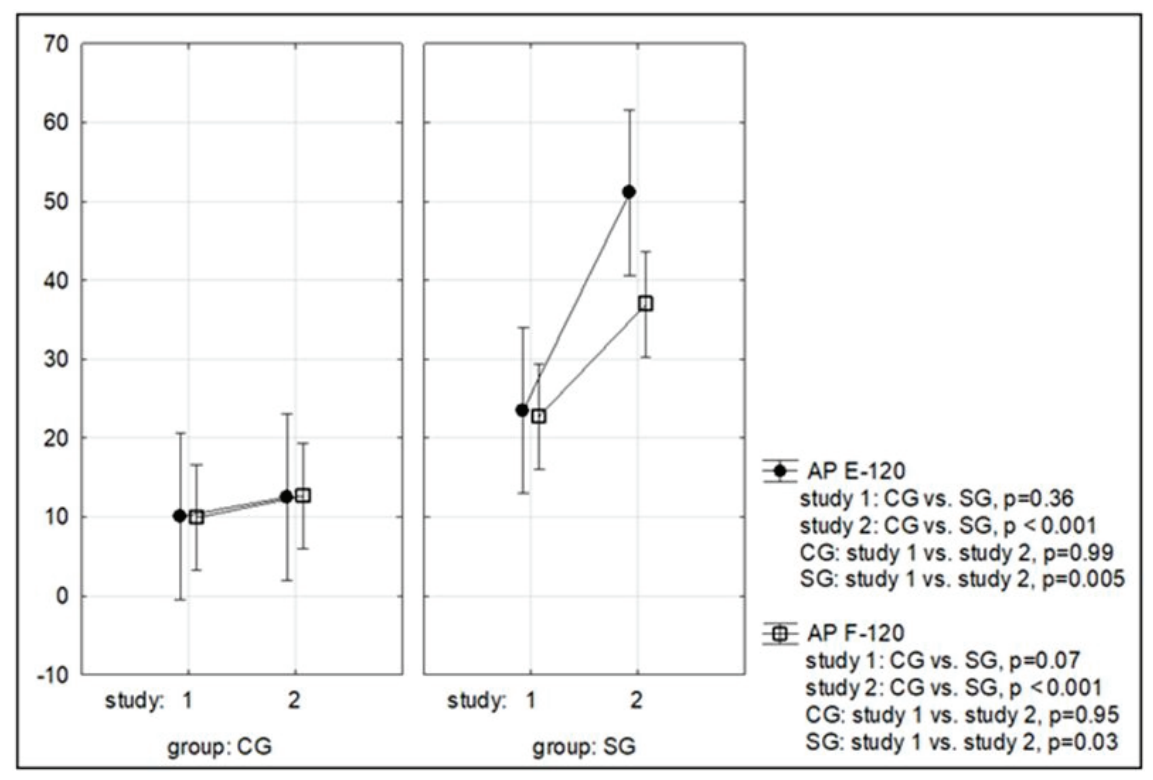

Fig. 4. Average Power [W] of the trunk muscles before and after the 8-week exercise programmes in the examined groups.

Values are expressed as mean (95\% confidence interval), $p$-values based on the Scheffe post-hoc test.

Abverations: CG - Control Group, SG - Study Group, AP - Average Power, E - trunk extensors, F - trunk flexors 


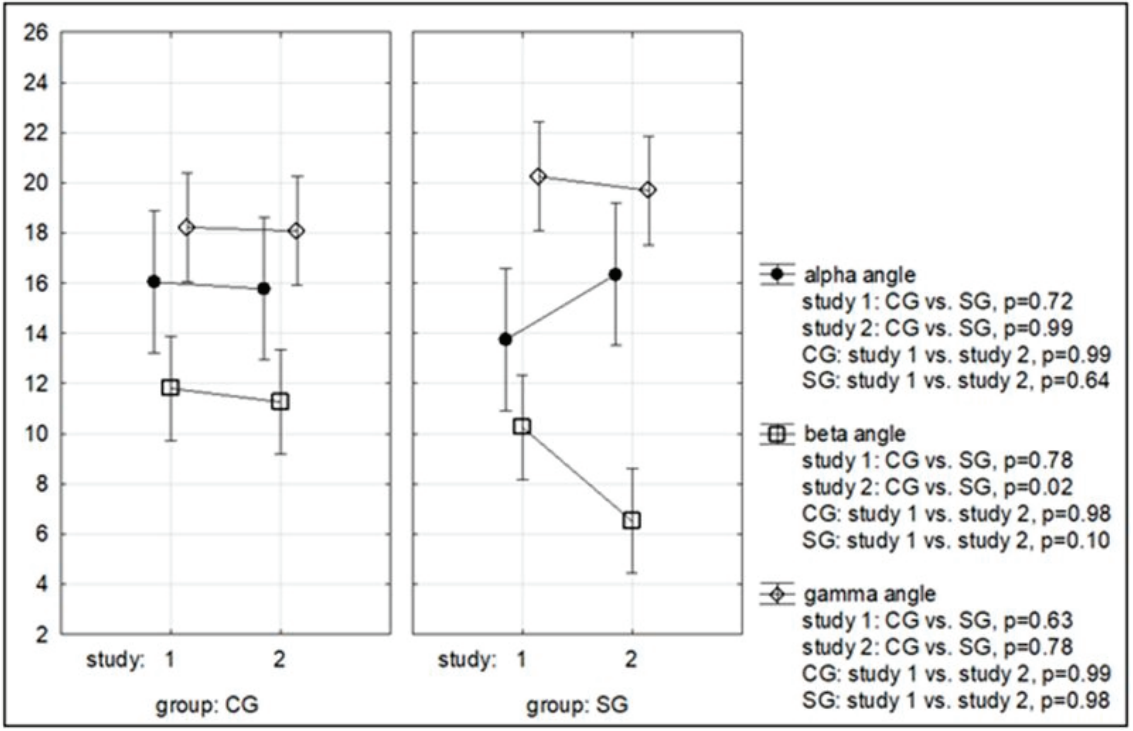

Fig. 5. Angular parameters $\left[^{\circ}\right]$ examining the sagittal spinal curvatures before and after the 8 -week exercise programmes in the examined groups. Values are expressed as mean (95\% confidence interval), $p$-values based on the Scheffe post-hoc test.

Abverations: CG - Control Group, SG - Study Group, Alfa - lumbosacral angle, Beta - thoracolumbar angle,

Gamma - upper thoracic angle, E - trunk extensors, $\mathrm{F}$ - trunk flexors

Table 4. The Pearson's correlation coefficient $(r)$ of functional-postural dependencies of the trunk muscles before and after the aquatic exercise programme $(p<0.05)$

\begin{tabular}{|c|l|c|c|c|c|}
\hline \multirow{2}{*}{$\begin{array}{c}\text { Body posture } \\
\text { parameters }\left[{ }^{\circ}\right]\end{array}$} & \multicolumn{2}{c|}{ Total Work [J] } & \multicolumn{2}{c|}{ Average Power [W] } \\
\cline { 2 - 6 } & E & $\mathrm{F}$ & $\mathrm{E}$ & $\mathrm{F}$ \\
\hline \multirow{3}{*}{ Test 1} & Alfa & $0.49^{*}$ & 0.42 & $0.47^{*}$ & 0.41 \\
\cline { 2 - 6 } & Beta & 0.14 & 0.05 & 0.11 & 0.08 \\
\cline { 2 - 6 } & Gamma & -0.37 & -0.40 & -0.38 & -0.40 \\
\hline \multirow{3}{*}{ Test 2} & Alfa & 0.25 & 0.16 & 0.20 & 0.19 \\
\cline { 2 - 6 } & Beta & 0.20 & 0.16 & 0.21 & 0.11 \\
\cline { 2 - 6 } & Gamma & $-0.49^{*}$ & $-0.48^{*}$ & $-0.51^{*}$ & $-0.51^{*}$ \\
\hline
\end{tabular}

Abverations: Alfa - lumbosacral angle, Beta - thoracolumbar angle, Gamma - upper thoracic angle, E - trunk extensors, F - trunk flexors, $*$ denotes significances.

Table 5. The Pearson's correlation coefficient $(r)$ of functional-postural dependencies of the trunk muscles before and after the land exercise programme $(p<0.05)$

\begin{tabular}{|c|l|c|c|c|c|}
\hline \multirow{2}{*}{$\begin{array}{c}\text { Body posture } \\
\text { parameters }\left[{ }^{\circ}\right.\end{array}$} & \multicolumn{2}{c|}{ Total Work [J] } & \multicolumn{2}{c|}{ Average Power [W] } \\
\cline { 3 - 6 } & E & F & E & F \\
\hline \multirow{3}{*}{ Test 1} & Alfa & -0.26 & -0.30 & -0.25 & -0.33 \\
\cline { 2 - 6 } & Beta & -0.06 & -0.08 & -0.09 & -0.16 \\
\cline { 2 - 6 } & Gamma & -0.15 & -0.11 & -0.11 & -0.08 \\
\hline \multirow{3}{*}{ Test 2 } & Alfa & -0.11 & -0.05 & -0.11 & 0.02 \\
\cline { 2 - 6 } & Beta & -0.15 & -0.37 & -0.15 & -0.23 \\
\cline { 2 - 6 } & Gamma & $-0.54^{*}$ & -0.37 & $-0.58^{*}$ & -0.43 \\
\hline
\end{tabular}

Abverations: Alfa - lumbosacral angle, Beta - thoracolumbar angle, Gamma - upper thoracic angle, E - trunk extensors, F - trunk flexors, $*$ denotes significances.
In the $\mathrm{CG}$, no statistically significant correlations were found before exercises commenced. After the completion of the LE programme functional-postural relationships did appear in the gamma angle, but only in relation to the trunk extensor muscles in the scope of total work $(r=-0.54)$ and average power $(r=-0.58)$ (Table 5).

\section{Discussion}

Maintaining a proper trunk muscle function is considered to be crucial for sustaining correct geometry of the spine curvatures [2], [12]. Changes in the involvement of the trunk muscles in spine stabilization do have an impact on postural disorders. It is believed that increased tension in the extensor muscles of the trunk, with a reduced function of flexor muscles, results in pelvic anteversion, and increased lumbar lordosis. Conversely, too strong abdominal muscles in relation to the weakened muscles of the back result in pelvic retroversion, flattening of the lumbar lordosis and a compensatory increase of the thoracic kyphosis.

Sinaki et al. [23] reported a statistically significant negative relationship between the extensor muscles of the trunk and the size of thoracic kyphosis, and a positive relationship with respect to the lumbar spine and sacral inclination. Stronger extensor muscles of the 
back, the lower thoracic curvature, but also the greater lumbar lordosis and sacral inclination were found. Therefore, the efficiency of the trunk extensor muscles appears to be an important determinant of posture in healthy women. However, strengthening of only this muscle group may lead to an increase in lumbar lordosis, which is not interpreted as a beneficial and desired phenomenon from a spinal biomechanics' point of view. This necessitates the development of appropriate functional-postural relations [23].

The results of our research suggest that $\mathrm{AE}$ allow the optimisation of the functional-postural condition both on the part of the trunk extensor and flexor muscles. After the completion of aquatic rehabilitation, there was a statistically significant decrease in the size of the gamma angle along with an increase in the activity of both muscle groups. In comparison, following exercises on land, significant functional-postural correlations were achieved along the same spine section only in the trunk extensor muscles.

$\mathrm{BC}$ treatment may be a cause of abnormal spinal curvatures which need the correction [15], [20]. However, postural patterns must be improved by an enhancement of functional parameters in the right proportions of muscular tension and posture habits [12]. To this end, the results of the current study show that the implementation of an aquatic rehabilitation environment has a greater effect compared to standard LE. AE have a positive effect on the specific muscle function due to the presence of differentiated motor patterns, compared to their onshore equivalents. In addition, the constant movement of the centre of gravity under the water's resistance increases the function of the trunk flexor and extensor muscles in order to maintain a standing position. The control of postural stability is a type of specific feedback from the nervous system to the surrounding environment and, therefore, must meet optimal control conditions by incorporating maximum efficiency with minimal energy expenditure in order to maintain a particular posture [15], [19], [24].

It was proved that backward gait, especially with the use of Thera-Band aquafins placed near the ankle joints of the lower limbs, increases activation of the trunk muscles. Based on the scientific reports [11], [17], [19], in our study, we have included to the exercise programme walking backward executed in both: the water and land environment. Due to the limited range of vision to the rear during this specific physical activity, there is a strong stimulation of proprioceptive sensation, which increases muscular stabilization of the head, trunk and pelvis, while deep muscles of the back, abdomen and buttocks become stimulated. Somatosensory information is considered the most important in the process of maintaining balance in a standing position, while the remaining visual and vestibular systems play a supporting role [17]. Similarly, superficial electromyography tests exert greater muscular activity when walking backwards compared to forward walking [11]. It has been shown that the transfer of such training to the aquatic environment creates additional resistance during the walk, so that under these conditions the backward gait is even more effective than the forward gait or walking on land. There was a significantly greater activity of the deep muscles of the spine and quadriceps of the thighs and tibias as well as of the cardiovascular response [19].

Apart from the normal functioning of the central nervous system, muscles are one of the basic links in the post-control system of body posture. This function depends both on the efficiency of energy metabolism of the muscles and the efficiency of the entire postural control system. Correct anatomical relations within the spine and associated structures (either indirectly or directly) depend on the physiological development rhythm, laws of statics and dynamics, as well as on environmental conditions, including the lifestyle of a human being [3], [12].

Differentiating the relationships between sagittal spinal curvatures and isokinetic trunk muscle endurance subjected to the type of physical activity used has revealed that, describing the formations of spine's anterior-posterior curvatures is a very difficult task due to the lack of physiological norms. It is considered, however, that these curvatures should be balanced and not too excessive. Reducing their size may cause strain, while excessive increase does lead to premature tissue wear in places of increased load [6], [15].

\section{Conclusions}

The AE generate a statistically significant functional-postural correlation of the trunk flexor and extensor muscles of the thoracic spine against the standard LE performed in the gym, which regulate these relations only in the extensor muscles of the trunk. Therefore, rehabilitation programmes for postural correction should take into account both the assessment of spinal curvatures and the functioning of the muscular system, as well as their mutual coexistence.

This research may be a valuable guidance to therapists and clinicians. Our results support that mu- 
tual functional-postural relationships are important indicators of both diagnostic values of musculoskeletal dysfunctions as well as determining suitable therapy programmes aiming to correct sagittal spinal curvatures in women after $\mathrm{BC}$ treatment.

\section{Disclosure policy}

The authors declare that there is no conflict of interest regarding the publication of this paper.

\section{References}

[1] ANDrade C.P., ZAMunÉR A.R., Forti M., TAMBuRÚS N.Y., SILVA E., Effects of aquatic training and detraining on women with fibromyalgia: controlled randomized clinical trial, Eur. J. Phys. Rehabil. Med., 2018, 55 (1), DOI: 10.23736/ S1973-9087.18.05041-4.

[2] Barbosa A.J., Amorim M.H., ZandonAdE E., Delaprane M.L., Evalution of body posture in women with breast cancer, Rev. Bras. Ginecol. Obstet., 2013, 35 (5), 215-20.

[3] Barczyk-Pawelec K., DziUbeK W., Piechura J.R., RożeK K., Correlations between somatic features, anteroposterior spinal curvatures and trunk muscle strength in school children, Acta Bioeng. Biomech., 2017, 19 (1), DOI: 10.5277/ ABB-00537-2015-03.

[4] BARCZyK-PAwelec K., SiPKo T., Active self-correction of spinal posture in pain-free women in response to the command "straighten your back", Women Health, 2017, 57 (9), DOI: 10.1080/03630242.2016.1243605.

[5] Bartels E.M., Juhl C.B., Christensen R., Hagen K.B., Danneskiold-Samsøe B., DagfinRud H., Lund H., Aquatic exercise for the treatment of knee and hip osteoarthritis, Cochrane Database Syst. Rev., 2016, 3, DOI: 10.1002/14651858.

[6] Ben Moussa Zouita A., Ben SAlah F.Z., DZiri C., BEARDSLEY C., Comparison of isokinetic trunk flexion and extension torques and powers between athletes and nonathletes, J. Exerc. Rehabil., 2018, 14 (1), DOI: 10.12965/jer.1835126.563.

[7] Cantarero-Villanueva I., Fernández-Lao C., Caro-Morán E., Morillas-Ruiz J., Galiano-Castillo N., DÍAZ-

-Rodríguez L., Arroyo-Morales M., Aquatic exercise in a chest-high pool for hormone therapy-induced arthralgia in breast cancer survivors: a pragmatic controlled trial, Clin. Rehabil., 2013, 27 (2), DOI: 10.1177/ 0269215512448256.

[8] Cantarero-Villanueva I., Fernández-lao C., Cuesta-Vargas A. I., Del Moral-Avila R., FernándeZ-DE-las-Peñas C., Arroyo-Morales M., The effectiveness of a deep water aquatic exercise program in cancer-related fatigue in breast cancer survivors: a randomized controlled trial, Arch. Phys. Med. Rehab., 2013, 94 (2), DOI: 10.1016/ j.apmr.2012.09.008.

[9] Cantarero-Villanueva I., Fernández-Lao C., FernándeZ-DE-Las-Peñas C., Lopez-Barajas I. B., Del-Moral-Ávila R., DE LA-Llave-Rincón A.I., ARroyo-Morales M., Effectiveness of water physical therapy on pain, pressure pain sensitivity, and myofascial trigger points in breast cancer survivors: a randomized, controlled clinical trial, Pain Med.
2012, 13 (11), DOI: 10.1111/j.1526-4637.2012.01481.x.

[10] Cuesta-Vargas A.I., Buchan J., Arroyo-Morales M., A multimodal physiotherapy programme plus deep water running for improving cancer-related fatigue and quality of life in breast cancer survivors, Eur. J. Cancer Care, 2014, 23 (1), DOI: 10.1111/ecc.12114.

[11] Croy T., Cross L., Cardiopulmonary responses to a backward walking maximal exercise test, Physioterapy Canada, 2002, 54 (1), 42-46.

[12] Elsayed W., Farrag A., Muaidi Q., Almulhim N., Relationship between sagittal spinal curves geometry and isokinetic trunk muscle strength in adults, Eur. Spine J., 2018, 27 (8), DOI: $10.1007 / \mathrm{s} 00586-017-5454-3$.

[13] Granacher U., Lacroix A., Roettger K., Gollhofer A., MUEHLBAUER T., Relationships between trunk muscle strength, spinal mobility, and balance performance in older adults, J. Aging Phys. Act, 2014, 22, DOI: 10.1123/japa.20130108.

[14] Guilhem G., Giroux C., Couturier A., Maffiuletti N.A., Validity of trunk extensor and flexor torque measurements using isokinetic dynamometry, J. Electromyogr. Kinesiol., 2014, 24 (6), DOI: 10.1016/j.jelekin.2014.07.006.

[15] Hanuszkiewicz J., Malicka I., BarCZYK-PAWEleC K., WOŹNIEWSKI M., Effects of selected forms of physical activity on body posture in the sagittal plane in women post breast cancer treatment, J. Back Musculoskelet Rehabil., 2015, 28, DOI: $10.3233 /$ BMR-140487.

[16] Kargarfard M., Shariat A., Ingle L., Cleland J.A., KARGARFARD M., Randomized controlled trial to examine the impact of aquatic exercise training on functional capacity, balance, and perceptions of fatigue in female patients with multiple sclerosis, Arch. Phys. Med. Rehabil., 2018, 99 (2), DOI: 10.1016/j.apmr.2017.06.015.

[17] LAUFER Y., Age and gender-related changes in the temporalspatial characteristics of forwards and backwards gait, Physiother. Res. Int., 2003, 8 (3), DOI: 10.1002/pri.281.

[18] Lindquist H., Enblom A., Dunberger G., Nyberg T., BERGMARK K., Water exercise compared to land exercise or standard care in female cancer survivors with secondary lymphedema, Lymphology, 2015, 48 (2), 64-79.

[19] Masumoto K., Takasugi S., Hotta N., Fujishima K., IWAMOTO Y., A comparison of muscle activity and heart rate response during backward and forward walking on an underwater treadmill, Gait Posture, 2007, 25 (2), DOI: 10.1016/ j.gaitpost.2006.03.013.

[20] Montezuma T., De Oliveira Guirro E.C., Leite M.M.O.L., VERNAL S., Changes in postural control in mastectomized women, J. Cancer Ther., 2014, 5(6), DOI: 10.4236/jct.2014.56056.

[21] RABElo M., FACHIN-MARTINS E., Inter-rater and test/retest reliabilities of the isokinetic measurements: assessing strength and endurance of the trunk muscles in two different protocols for able-bodied and post-stroke hemiparesis, Top Stroke Rehabil., 2018, 25(26), DOI: 10.1080/ 10749357.2018.1481568

[22] Schmitz K.H., Courneya K.S., Matthews C., Demark-Wahnefried W., Galvão D.A. et al., American College of Sports Medicine roundtable on exercise guidelines for cancer survivors, Med. Sci. Sports Exerc., 2010, 42 (7), DOI: 10.1249/MSS.0b013e3181e0c112.

[23] Sinaki M., Itoi E., Rogers J.W., Bergstralh E.J., WAHNER H.W., Correlation of back extensor strength with thoracic kyphosis and lumbar lordosis in estrogen-deficient women, Am. J. Phys. Med. Rehabil., 1996, 75 (5), DOI: 
10.1097/00002060-199609000-00013.

[24] TAKeshima N., Rogers M.S., Watanabe E., Brechue W.F., OKADA A., YAMADA T., ISLAM M.M, HAYANO J., Waterbased exercise improves health-related aspects of fitness in older women, Med. Sci. Sports Exerc., 2002, 34 (3), 544-551.
[25] Tsourlou T., BeniK A., Dipla K., Zafeiridis A., Kellis S., The effects of twenty-four-week aquatic training program on muscular strength performance in healthy elderly women, J. Strength Cond. Res., 2006, 20 (4), 811-818. 